\title{
On the Determination of the Crossing-Point Temperatures for Evaluating Reactivity of Solids
}

\author{
YIN WANG, HAIHUI WANG, SHENGNAN SONG, and NA MENG \\ State Key Laboratory of Fire Science \\ University of Science and Technology of China \\ 96 Jinzhai Road, Hefei \\ PR China 230026
}

\begin{abstract}
In this paper, we study the physical basis of the crossing-point temperature (CPT) techniques and seek guidelines for attaining reliable evaluation on the kinetic parameters of the exothermic reactions that occurred during the self-heating of solids. By numerically simulating the thermal response of a solid sample placed in a convective thermal environment, the variation in the trend of the enthalpy change term together with the heat generation term in the sample central elementary volume was monitored, which allowed a close observation on the formation of a CPT. It was found that a true CPT is the transition temperature at which the heat transfer from the surroundings to the central neighborhood goes into reverse. The CPT values measured during practical testing may be altered by experimental settings for finding equal temperatures between the sample centre and a reference point, which is more evident at the conditions of enlarged sample sizes and elevated oven temperatures. Relaxation on the criterion of the equality between the enthalpy change term and the heat generation term in the central elementary volume yielded a theoretical correlation for determining the maximum distance for the reference temperature sensor to be placed in order to perform reliable measurement of a CPT. This correlation is in excellent agreement with the experimental settings for the reference point adopted by various research groups.
\end{abstract}

KEYWORDS: solid, self heating, spontaneous ignition, crossing-point temperature.

\section{NOMENCLATURE LISTING}

\begin{tabular}{|c|c|c|c|}
\hline$A$ & pre-exponential factor $(1 / \mathrm{s})$ & $T$ & temperature $(\mathrm{K})$ \\
\hline$C_{p}$ & specific heat capacity $(\mathrm{J} / \mathrm{kg} \cdot \mathrm{K})$ & $T_{0}$ & sample initial temperature $(\mathrm{K})$ \\
\hline E & apparent activation energy of the & $T_{a}$ & oven temperature $(\mathrm{K})$ \\
\hline & exothermic reactions $(\mathrm{J} / \mathrm{mol})$ & $T_{p}$ & crossing-point temperature $(\mathrm{CPT})(\mathrm{K})$ \\
\hline$h$ & coefficient of convective heat transfer & Greek & \\
\hline & $\left(\mathrm{W} / \mathrm{m}^{2} \cdot \mathrm{K}\right)$ & & dimensionless radius, $r / r_{0}$ \\
\hline & thermal conductivity $(\mathrm{W} / \mathrm{m} \cdot \mathrm{K})$ & $\eta_{m}$ & dimensionless maximum distance \\
\hline lc & heat conduction term & $\eta_{r}$ & dimensionless distance of a reference \\
\hline$q_{s}$ & heat generation term & & point \\
\hline$q_{t}$ & enthalpy change term & $\theta$ & dimensionless temperature \\
\hline$Q$ & heat of exothermic reactions $(\mathrm{J} / \mathrm{kg})$ & $\theta_{p}$ & dimensionless crossing-point \\
\hline & radius $(\mathrm{m})$ & & temperature \\
\hline$r_{0}$ & sample/reactor radius (m) & $\rho$ & density $\left(\mathrm{kg} / \mathrm{m}^{3}\right)$ \\
\hline & maximum distance for placing a & $\tau$ & dimensionless time, $t / \tau_{c}$ \\
\hline & reference temperature sensor $(\mathrm{m})$ & $\tau_{c}$ & characteristic reaction time \\
\hline & universal gas constant $(\mathrm{J} / \mathrm{mol} \cdot \mathrm{K})$ & $\tau_{d}$ & characteristic diffusion time \\
\hline
\end{tabular}

\section{INTRODUCTION}

Coal and some other solids can react with oxygen in open atmosphere. Such reactions are exothermic and may eventually lead to the self-heating as well as spontaneous combustion of the solids. For this reason, these reactive solids are categorized as 'pyrophoric' materials, and are regularly evaluated by their propensity towards self-heating and spontaneous combustion for the purpose of their safe utilization and handling [1-3]. 
The crossing-point temperature techniques introduced in last two decades are known as major methods for determining the reactivity of solids [2,3]. Derived from the conventional approach [3], these techniques have made it possible to evaluate the apparent kinetic data of the exothermic reactions that occur during the self-heating and spontaneous combustion of solids. These techniques have two versions in principal, with one developed by Chen's research group in the middle of the 1990s [4-7]. The kernel of Chen's CPT technique is to find out the time interval when the temperature at the centre of a sample is equal to that at an adjacent position by heating the sample in an oven at a constant temperature. At this time interval, the energy conservation equation in the central elementary volume can be simplified into equality between the enthalpy change term and the heat generation term. The crossing-point temperatures and their associated rates of temperature rise measured at different oven temperatures are then used to form a plot of $\ln \left(\partial T_{p} / \partial t\right)$ against the reciprocal of the crossing-point temperature $T_{p}$, which yields the evaluation of the kinetic parameters of exothermic reactions that occurred during the self-heating of the solid. The other version was introduced by Jones in the later part of the 1990s [8-11]. Its testing procedure is identical to that of Chen's method. The only difference between these two methods is that in Jones method the oven temperature is set as the reference temperature for determining both the CPT and the associated time derivative $\left(\partial T_{p} / \partial t\right)$.

Compared to the basket heating method based upon the Frank-Kamenetskii model, these crossing-point temperature techniques have obvious advantages in providing a quick assessment of the apparent kinetic parameters of the exothermic reactions of solids $[2,3]$. The advantages include the shortened duration of a single test and no necessity to run measurements with various sample sizes, which have been confirmed by comparative measurements carried out by independent research groups with the solids such as milk powder, wood sawdust and coal [5-7,12-15]. However, for a long time, issues have been raised on the CPT techniques due to the variation in the setting of a reference point, which are partly reflected in the disputes among the developers of the individual techniques [16-19]. As reported in the literature [7], the crossing-point temperature determined by Chen's technique departs from the oven temperature, with a variation ranging from several to a few tens of kelvin. The extent of departure depends on the temperature of the oven and the properties of the sample tested. These two techniques are almost identical in nature, but yield different test results even under the same experimental conditions. The obvious question is which results are more reliable. Generally speaking, in the utilization of the CPT techniques there are two basic impending issues: 1) how do the temperature of the crossing-point and its corresponding rate of temperature rise relate to the kinetics of the exothermic reactions and 2) in which way does the crossing-point temperatures measured contain essential information for reliable determination of the kinetic parameters in association with the reactivity of solids.

Attempts are made in the present paper to find out the answers to the above issues. Based upon the energy conservation equation, the nature of the crossing-point temperature and its physical significance are investigated in detail. The relationship between the CPT and the solid properties as well as the experimental parameters is studied using a sensitivity analysis technique. By setting various distances for the reference point for determining the CPTs, the variation in the CPTs and the consequent results of the kinetic parameters are simulated, and the difference between the Chen's and Jones' techniques is then analyzed in depth. Finally, general principles are also explored for setting the distance of the reference point to achieve reliable measurement of a CPT.

\section{THE MODEL AND NUMERICAL METHOD}

A sample consisting of fine solid particles is placed in a shaped metal basket and is heated in a convective environment at a constant temperature $T_{a}$. Temperature rise of the sample triggers the exothermic reactions occurring inside it. The sample is considered to be homogeneous and isotropic everywhere. The reactions are single-step ones with the rate expression being independent of the reactant concentrations and obeying the Arrhenius law. By neglecting heat effects of gas diffusion within the sample, the energy balance equation for an elementary volume at any position can be written by

$\rho C_{p} \frac{\partial T}{\partial t}=k\left(\frac{\partial^{2} T}{\partial r^{2}}+\frac{j}{r} \frac{\partial T}{\partial r}\right)+\rho Q A \exp \left(-\frac{E}{R T}\right)$ 
where $j$ is the shape factor, and $j=0,1$ or 2 is for a slab, a cylinder or a sphere, respectively [1]. The left-hand side of the equation corresponds to the enthalpy change term in an elementary volume, while the first term at the right-hand side represents the heat conduction term and the second term is the heat generation term. Effect of moisture content of the sample on the energy balance is not considered in Eq. 1, since the temperature range studied exceeds the boiling point of water $[1,2]$.

The initial condition for Eq. 1 is:

$T=T_{0}, \quad$ at $t=0$

The central symmetry and convective boundary conditions are given by

$$
\begin{array}{ll}
\frac{\partial T}{\partial r}=0, & \text { at } \quad r=0 \\
-k \frac{\partial T}{\partial r}=h\left(T-T_{a}\right), & \text { at } \quad r=r_{0}
\end{array}
$$

By introduction a dimensionless temperature $\theta=\frac{E}{R T_{0}^{2}}\left(T-T_{0}\right) \quad[1,20]$, we have

$-\frac{E}{R T}=-\frac{E}{R T_{0}}+\frac{\theta}{1+\varepsilon \theta}$

where $\varepsilon=\frac{R T_{0}}{E}$, and its value is far less than unity.

For a long cylindrical sample, the dimensionless energy balance equation is then written by

$\frac{\partial \theta}{\partial \tau}=\frac{\tau_{c}}{\tau_{d}} \frac{1}{\eta} \frac{\partial}{\partial \eta}\left(\eta \frac{\partial \theta}{\partial \eta}\right)+e^{f(\theta)}$

where $f(\theta)=\frac{\theta}{1+\varepsilon \theta}, \quad \tau_{c}=\frac{R T_{0}^{2} C_{p}}{E Q A \exp \left(-\frac{E}{R T_{0}}\right)}$, and $\tau_{d}=\frac{\rho C_{p} r_{0}^{2}}{k}$ [20].

Initial condition is re-written as

$\theta=0, \quad$ at $\quad \tau=0$

and the boundary conditions are

$\frac{\partial \theta}{\partial \eta}=0, \quad$ at $\quad \eta=0$ 
$-\frac{\partial \theta}{\partial \eta}=\frac{h r_{0}}{k} \theta+\frac{h r_{0} E}{k R T_{0}^{2}}\left(T_{0}-T_{a}\right), \quad$ at $\quad \eta=1$

The equation with the initial and boundary conditions (Eqs. 6-9) were discretized and solved numerically using the finite volume method. The integrals of the enthalpy change and heat generation terms were approximated using trapezoidal quadrature rule, while the spatial derivatives of the thermal diffusion term were expanded using one-step central difference. Time integration was carried out under the implicit scheme. The algebraic equations generated were solved using the Gauss-Seidel iteration technique. The programming was performed in Visual Fortran 6.5, and the time step was set at $0.1 \mathrm{~s}$ for all calculations. Temperature histories at the sample centre were determined first, and the crossing-point temperature was then found by placing a reference point and tracing the time interval when their temperatures were met. The rate of temperature rise at the crossing point was computed following the rule of 5-point forward difference.

\section{RESULTS AND DISCUSSION}

\section{Significance of the Crossing-Point Temperatures and their Dependence to the Experimental Parameters}

Firstly, we consider a theoretical CPT at which the enthalpy change term $q_{t}$ equals to the heat generation term $q_{s}$ in the central elementary volume for a reactive solid sample placed in a steady heating environment. By numerically simulating the temperature rise at the sample centre and the associated time derivative, variation in the magnitudes of $q_{t}$ and $q_{s}$ is traced, which allows an observation of the status of $q_{t}=q_{s}$. Values of the parameters adopted in calculation are listed in Table 1.

Table 1. Evaluation of the parameters utilized in the present calculations.

\begin{tabular}{lll}
\hline Parameter & ${\text { Solid } \mathbf{A}^{\mathbf{a}}}$ & Solid B $^{\mathbf{b}}$ \\
\hline$\rho\left(\mathrm{kg} / \mathrm{m}^{3}\right)$ & 725 & 1350 \\
$C_{p}(\mathrm{~J} / \mathrm{kg} \cdot \mathrm{K})$ & 1240 & 1132 \\
$k(\mathrm{~W} / \mathrm{m} \cdot \mathrm{K})$ & 0.11 & 0.11 \\
$E(\mathrm{~kJ} / \mathrm{mol})$ & 90.0 & 125.1 \\
$Q A(\mathrm{~J} / \mathrm{kg} \cdot \mathrm{s})$ & $4.48 \times 10^{12}$ & $2.73 \times 10^{17}$ \\
$h\left(\mathrm{~W} / \mathrm{m}^{2} \cdot \mathrm{K}\right)$ & $14^{\mathrm{c}}$ & 14 \\
\hline
\end{tabular}

${ }^{a}$ The sample of the Prima coal utilized by Nugroho and co-workers [13];

${ }^{\mathrm{b}}$ The sample of 0.5 M KAc-ion-exchanged coal [14];

${ }^{\mathrm{c}}$ The value recommended by Gray and Halliburton [2].

Figure 1 demonstrates the typical variation of the enthalpy change term as well as the heat generation term in the central elementary volume for Solid A being placed in an environment at different temperatures. It can be seen that, at the early stage of the temperature rise, the magnitude of the enthalpy change term rises rapidly and then drops after reaching a peak. Meanwhile, for the heat generation term, its value increases slowly from unity. At the later stage, the increased value of the heat generation term and the decreased value of the enthalpy change term lead to these two terms intersecting and then separate from one another. Distinct trends are shown for the curves in Figs. 1 $\mathrm{a}$ and $\mathrm{b}$ after the appearance of an intersection, with the former ones undergoing slow progress and the later ones going up sharply. This is indicative that, in the first case, the sample may never reach the status of spontaneous combustion after the temperature preparation at the early stage, while in the second case, spontaneous combustion eventually occurs within the sample after the intersection. In both scenarios, the curves only meet once at the temperatures $\theta_{p}$ equal to 11.03 and 14.28, respectively. Nugroho and co-workers [13] conducted measurements on the same solid and found the values of $\theta_{p}$ in a range of between 9.92 and 14.46 under various oven temperatures. Since 
there was a lack of information of the oven temperatures for every $\theta_{p}$, it was not possible to compare their test results with the present predictions.

Once a sample is placed in an oven, heat is transferred by conduction from its surface to the centre. Under this circumstance, the heat generation term is negligible due to low initial temperature at the sample centre, and the heat conduction term plays major role in raising the enthalpy in the central elementary volume. With an increase in the temperature at the sample centre, there is a reduction in the temperature difference between the sample centre and its neighbor due to the activation of the heat generation term. A specific time interval may be reached when the increased value of heat generation term is just equal to that of the enthalpy change term in the central elementary volume. After this time interval, the heat generation term undergoes progressive increase, allowing the sample to be in a quasi-steady state, as exhibited in Fig. 1a. Otherwise, significant increase in the heat generation term may eventually lead to the spontaneous combustion of the sample (Fig. 1b). Under particular circumstances, the time interval may never be reached for equality between the heat generation term and the enthalpy change term within the sample central elementary volume. This occurs once the temperature at a position outside the central elementary volume firstly surpasses the critical temperature for initiating spontaneous combustion.

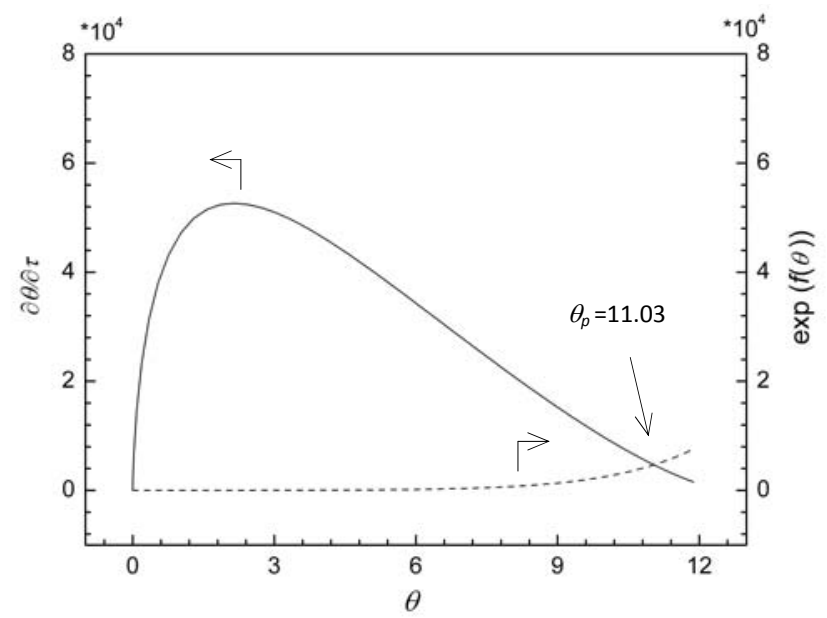

(a)

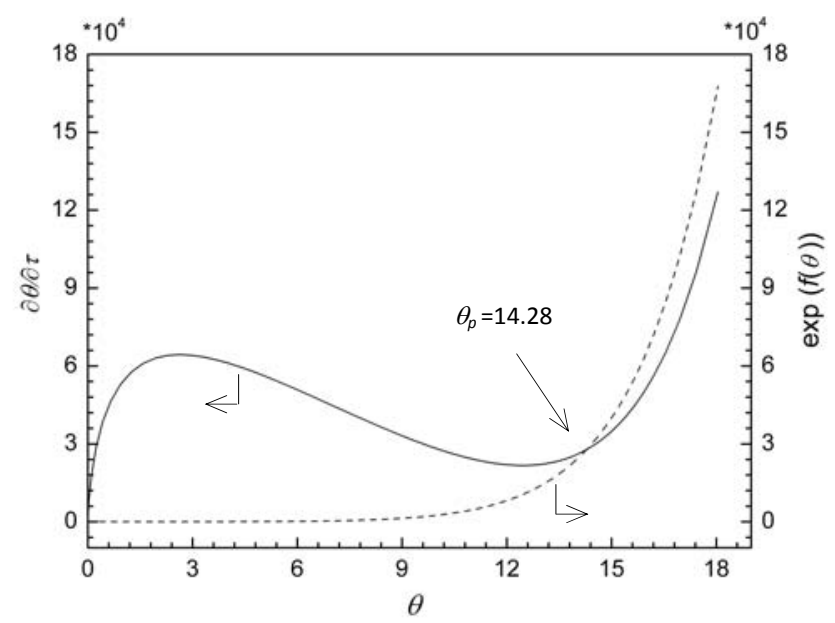

(b)

Fig. 1. Variation of the terms $\partial \theta / \partial \tau$ (dimensionless enthalpy change term) and $\exp (f(\theta))$ (dimensionless heat generation term) at the sample centre as a function of the parameter $\theta$ for Solid A with a sample radius of $25 \mathrm{~mm}$. In the calculations two distinct oven temperatures are considered: (a) $T_{a}=390 \mathrm{~K}$; (b) $T_{a}=410 \mathrm{~K}$. 
The phenomenon of the crossing point can be further interpreted based upon the energy conservation equation at the central neighborhood. After an arrangement, Eq. 6 can be integrated in the following form

$$
\int_{0}^{2 \pi} \int_{0}^{\Delta \eta} \eta \frac{\partial \theta}{\partial \tau} d \eta d \varphi=\int_{0}^{2 \pi} \int_{0}^{\Delta \eta} \frac{\tau_{c}}{\tau_{d}} \frac{\partial}{\partial \eta}\left(\eta \frac{\partial \theta}{\partial \eta}\right) d \eta d \varphi+\int_{0}^{2 \pi} \int_{0}^{\Delta \eta} \eta e^{f(\theta)} d \eta d \varphi
$$

For the sample owning homogeneous and isotropic properties, once $\Delta \eta$ is small enough, the temperatures and their associated time derivatives are evenly distributed within the central elementary volume. Thus, Eq. 10 can be casted into

$$
\left.\pi(\Delta \eta)^{2} \frac{\partial \theta}{\partial \tau}\right|_{\eta=\Delta \eta}=2 \pi \frac{\tau_{c}}{\tau_{d}}\left[\left.\left(\eta \frac{\partial \theta}{\partial \eta}\right)\right|_{\eta=\Delta \eta}-\left.\left(\eta \frac{\partial \theta}{\partial \eta}\right)\right|_{\eta=0}\right]+\left.\pi(\Delta \eta)^{2} e^{f(\theta)}\right|_{\eta=\Delta \eta}
$$

An utilization of the axial symmetry conditions at $\eta=0$, the above equation is then further simplified to

$$
\left.\frac{2}{\Delta \eta} \frac{\tau_{c}}{\tau_{d}} \frac{\partial \theta}{\partial \eta}\right|_{\eta=\Delta \eta}=\left.\frac{\partial \theta}{\partial \tau}\right|_{\eta=\Delta \eta}-\left.e^{f(\theta)}\right|_{\eta=\Delta \eta}
$$

As shown in Fig. 1, prior to the appearance of the crossing point, the enthalpy change term is larger than the heat generation term, i.e. $q_{t}>q_{s}$; thus, we have $\left.\frac{\partial \theta}{\partial \eta}\right|_{\eta=\Delta \eta}>0$. This is an indication of the conduction of heat from outside to the sample centre at this stage. At the crossing-point temperature, there is $\left.\frac{\partial \theta}{\partial \eta}\right|_{\eta=\Delta \eta}=0$ due to the equality between the enthalpy change term and the heat generation term. Afterwards, we have $q_{t}<q_{s}$, and hence $\left.\frac{\partial \theta}{\partial \eta}\right|_{\eta=\Delta \eta}<0$, indicating that the heat begins to transfer from the central elementary volume to the outside through conduction.

It is evident that the crossing-point temperature is the transition temperature at which heat conduction from the outside to the central elementary volume goes into reverse. At this particular time interval, the adiabatic conditions are attained at the $\Delta \eta$ interface, and within the interface there is a direct balance between the enthalpy change term and the heat generation term. After that, the magnitude of the enthalpy change term increases continuously as a result of the intensified exothermic reactions (the heat generation term) and the heat conduction term emerges again (refer to Fig. 1). There is no doubt that for a reactive solid undergoing self-heating and spontaneous combustion, the status of $q_{t}=q_{s}$ may only occur once.

From the perspective of the governing equation for energy, the temperature of the sample centre and the corresponding rate of temperature rise at the time interval of vanishing heat conduction term should not only be a function of solid reactivity and thermal properties, but also be influenced by the experimental conditions. Using a 'brute force' method, the sensitivity of $T_{p}$ and $\partial T_{p} / \partial t$ to several parameters is analyzed. Figure 2a demonstrates how the crossing-point temperature is altered by enlarging or reducing the magnitude of each parameter at a level of $\pm 10 \%$ of the reference value. It is evident that the parameters that have significant influence on $T_{p}$ are the apparent activation energy $E$ and the oven temperature $T_{a}$. For a sample with a reduced apparent activation energy $E$, the rate of heat generation increases more considerably with the temperature rise at its centre. A higher temperature $T_{p}$ is then required to allow commencing the status of vanishing heat conduction term in the sample's central elementary volume. Effect of the oven temperature on $T_{p}$ may operate in a similar way, by altering the temperature rise at the sample's centre and 
hence the rate of heat generation in the central elementary volume.

Effects of the parameters on the rate of temperature rise at the crossing point are shown in Fig. 2b. For convenience in data comparison, logarithm of the rate of temperature rise is used here. It is observed that the influence of the apparent activation energy and the oven temperature on the rate of temperature rise at the crossing point far exceeds that of other parameters. This is readily understandable, if we recall the criterion for finding the CPTs, that is, an equality between the enthalpy change term $(\partial \theta / \partial \tau)$ and the heat generation term $(\exp (f(\theta)))$ in the central elementary volume of a sample. In the CPT measurements, the crossing-point temperatures and their associated rates of temperature rise are essentially governed by the reactivity of the solids and the set oven temperatures. This feature essentially ensures the reliability and effectiveness of the CPT techniques in determining the activation energy and heat of reactions of the solids.

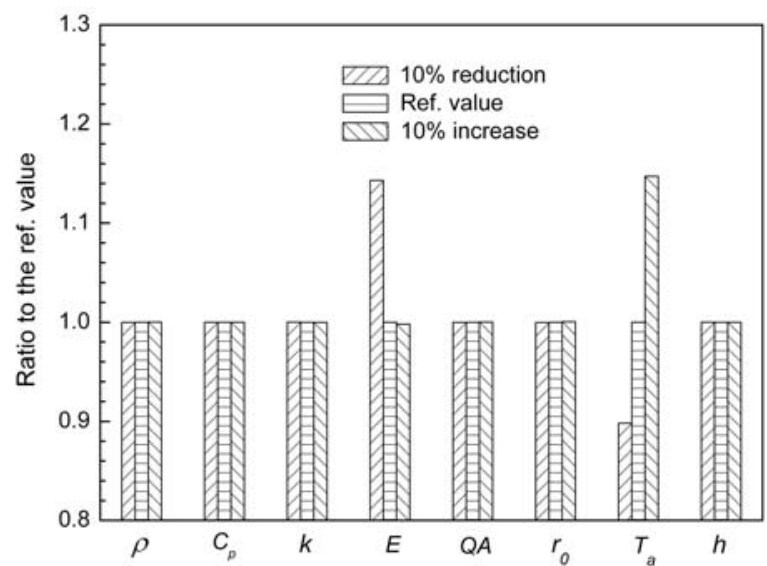

(a)

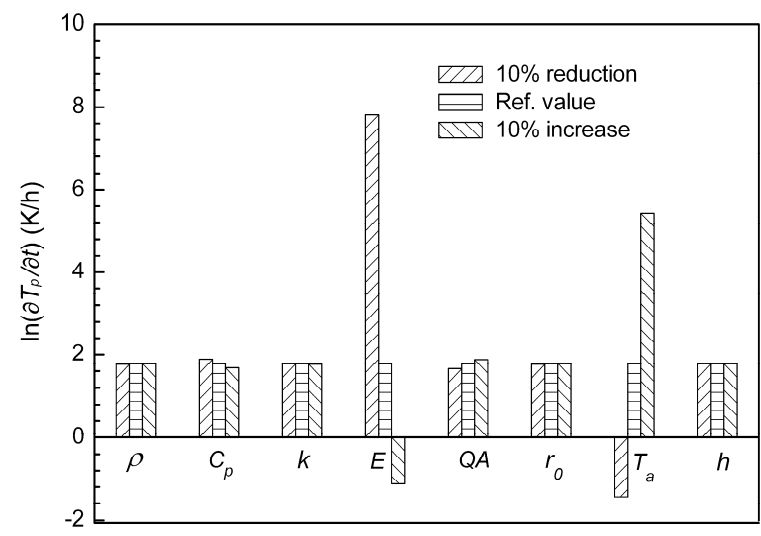

(b)

Fig. 2. Analysis on the sensitivity of the crossing-point temperature (a) and the corresponding rate of temperature rise (b) to the experimental parameters. The calculations were done for Solid A by considering the reference sample radius of $25 \mathrm{~mm}$ and the oven temperature of $380 \mathrm{~K}$.

\section{Variation in the CPTs Determined by Placing the Reference Point at Varying Distances}

In practice, a CPT is determined by monitoring the temperature rise both at the sample's centre and at a reference point as previously mentioned. In Chen's method [4,5], the reference point is suggested at a position several millimeters away from the centre, and the CPT is found when the central temperature is equal to that of the reference point. In the Jones' method [8,9], oven temperature is set as a reference. This setting is basically equivalent to taking the sample's surface temperature as the reference, since for a sample heated in a convective environment its surface temperature is rather close to the oven temperature 
once the temperature at the sample centre reaches that at the surface [21,22]. One more variation in determining the CPTs is the so-called Nordtest method [2], with the reference point being placed in the middle of the sample radius. Now, we examine how the CPTs are affected by the distances adopted for placing the reference point.

Figures $3 \mathrm{a}$ and $\mathrm{b}$ show the subtraction of the crossing-point temperature from the oven temperature as a function of dimensionless distance $\eta_{r}$ set for placing a reference point under specific circumstances. It can be seen that, for a lower oven temperature $T_{a}, T_{p}-T_{a}$ does not vary with $\eta_{r}$ significantly; however, for an elevated oven temperature $T_{a}$, the magnitude of $T_{p}-T_{a}$ is prominently altered by $\eta_{r}$. Similarly, at the condition of the same oven temperature, a smaller sample radius $r_{0}$ leads to relatively-flat distribution of the term $T_{p}-T_{a}$, while an enlarged sample radius $r_{0}$ may result in significant variation in the term $T_{p}-T_{a}$ with the distance $\eta_{r}$. These typical phenomena are in consistent with the observations obtained during the practical CPT measurements using both Chen's and Jones' methods [7]. During the self-heating experiments with bagasse and some other materials in the constant temperature environment, Griffiths and co-workers [21] found that the temperature difference between the sample centre and the heating environment is usually above $25 \mathrm{~K}$ and becomes larger for an increased $r_{0}$. In contrast, the temperature difference between the sample surface and the heating environment remained at a level of $\sim 5 \mathrm{~K}$.

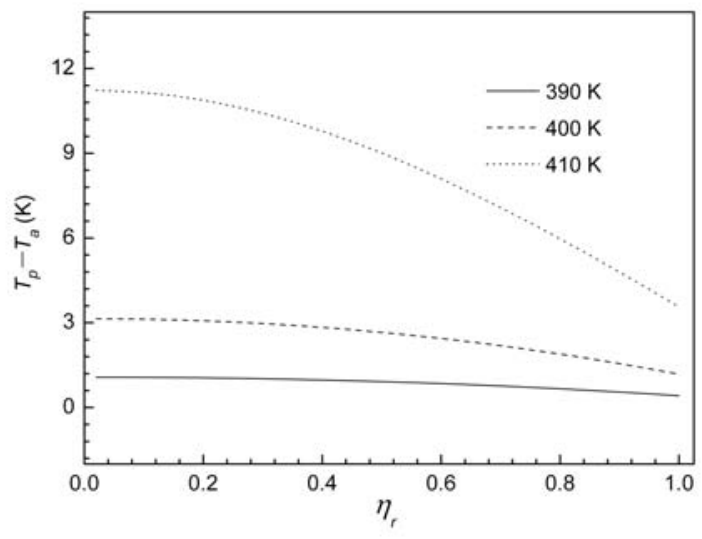

(a)

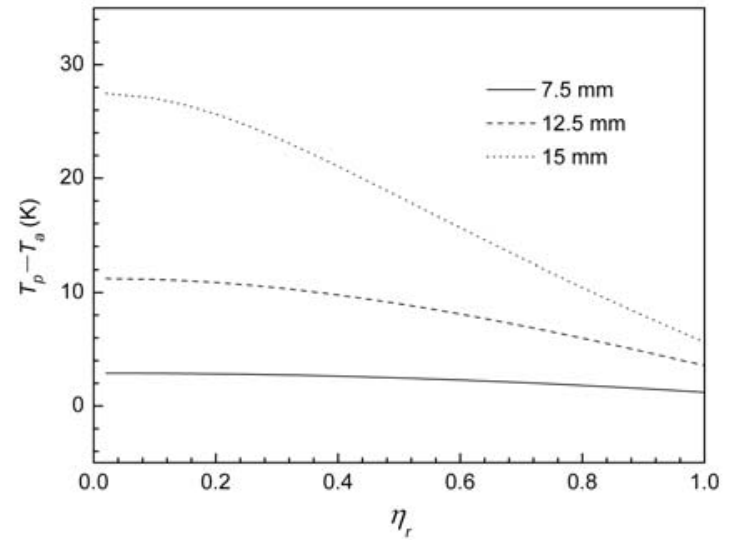

(b)

Fig. 3. Subtraction of the crossing-point temperature from the oven temperature as a function of dimensionless distance $\eta_{r}$ set for placing a reference point. The calculations were done for Solid B by taking into account: (a) a constant sample radius of $12.5 \mathrm{~mm}$; (b) the constant oven temperature of $410 \mathrm{~K}$.

Once a sample is placed in a convective heating environment, the temperature rise starts from its surface. Thermal diffusion process triggers the heat released by the localized heat generation term, creating wavy distribution of the temperatures across the sample radius. Once the peak temperature moves to the sample centre, the direction of heat conduction in the central elementary volume turns to reverse. This is the reason to make the CPT maintain at a relatively-high level, i.e., a considerable value of $T_{p}-T_{a}$. This phenomenon becomes more eminent, especially when $T_{a}$ is larger as a result of its direct impact to the localized heat generation term. As $r_{0}$ increases, the peak temperature may take a longer time to diffuse into the sample centre via conduction, which allows more heat accumulated locally as a result of the contribution of heat generation term in an extended period. For this reason, the peak temperature may be increased and hence the crossing-point temperature at the sample centre. It has often been observed during the practical testing that, for a relatively lower $T_{a}$ and a smaller $r_{0}$, the CPT results collected via Chen's and Jones' methods are getting close [7]. These trends are also in agreement with the results shown in Figs. 3a and b.

Distinct settings in the distance of a reference point introduced by individual test methods lead to the variation in determining the CPTs and the values of the term $\partial T_{p} / \partial t$, which inevitably affects the further evaluation of parameters $E$ and $Q A$. Figures $4 \mathrm{a}$ and $\mathrm{b}$ illustrate the distribution of the absolute relative errors of the parameters $E$ and $Q A$ as a function of the distance set for the reference point to the sample centre. It can be readily seen that the relative errors of $E$ and $Q A$ are very close to zero for the reference point located 
in the region of $\eta_{r} \leq 0.2$. This observation further confirms the reliability of the Chen's method, if we exclude the errors possibly induced by the practical measurements of experimental parameters. With the departure of the reference point from the sample centre, the relative errors increase significantly, especially for the term $Q A$. For $\eta_{r}=1.0$ (which is equivalent to the Jones' method), the errors for $E$ maintain at a level of $<1 \%$ but $\sim 50 \%$ for the term $Q A$. An error of less than $1 \%$ is negligible, and the considerable deviation in $Q A$ value comes from the technique for determining this term. By doing the measurements using both Chen's and Jones' methods, Nugroho and co-workers [12] reported that the apparent activation energy for South Bangko coal is $78 \pm 7$ and $75 \pm 4 \mathrm{~kJ} / \mathrm{mol}$, respectively, while for the KPC-Prima coal, the obtained value is $56 \pm 4$ and $54 \pm 2 \mathrm{~kJ} / \mathrm{mol}$, respectively. The closeness of test results for $E$ is direct evidence to support our findings.

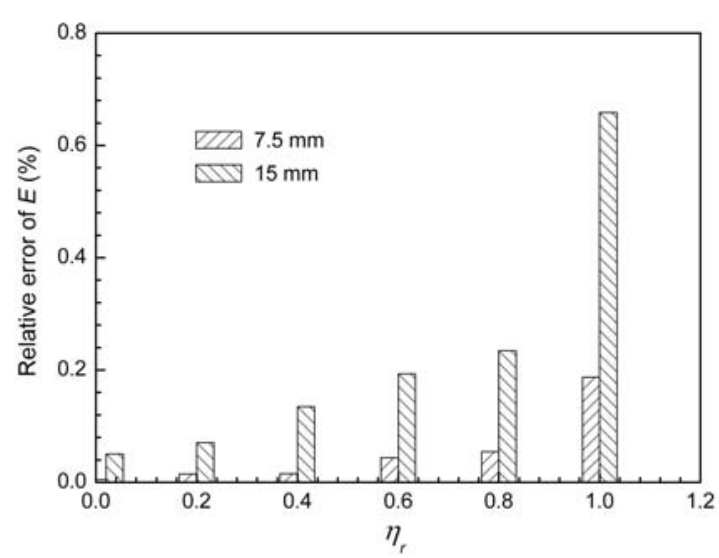

(a)

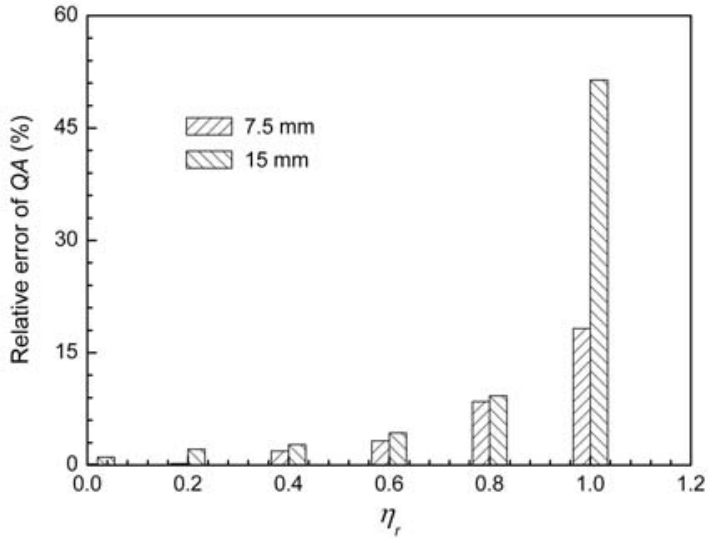

(b)

Fig. 4. Distribution of the absolute relative errors of the parameters: (a) $E$; (b) $Q A$ as a function of dimensionless distance of the reference point to the sample centre. The calculations were done for Solid B by considering a series of oven temperatures from 390 to $410 \mathrm{~K}$.

The reason behind the diversity in the values determined for $E$ and $Q A$ can be explored by comparing the enthalpy change term $q_{s}$ with the heat generation term $q_{t}$ in the sample's central elementary volume at the conditions of varying distances of the reference point. Figure 5 shows the variation of the ratio $q_{s} / q_{t}$ determined as a function of the distance set for the reference point. When the dimensionless radius $\eta_{r}$ is small, especially $\eta_{r}=0.02$, there exists $q_{s} / q_{t} \approx 1$. Under these situations, the CPTs determined can be regarded as the true temperature at which thermal conduction term vanishes in the central elementary volume. With the increase of $\eta_{r}$, there is $q_{s} / q_{t}<1$, indicating that the thermal conduction term is no longer negligible. This phenomenon is manifested at $\eta_{r} \rightarrow 1.0$.

The above observations were further confirmed by the temperature profile within a sample determined at the time when the identical temperature was reached between the sample centre and a reference point. As shown in Fig. 6, the temperature profile exhibits distinct patterns at the CPTs determined. For a small value of $\eta_{r}$, the temperatures are essentially indistinguishable within the region of $\eta \leq 0.2$, which corresponds to the situation of $q_{s} / q_{t} \approx 1$ in Fig. 5. When $\eta_{r}$ becomes larger, the temperature fluctuations become evident within the region of $\eta \leq \eta_{r}$, indicating obvious heat conduction occurring at the interface $\eta=\eta_{r}$. In the case of $\eta_{r}=1.0$, although there is equality between the surface temperature and the central temperature, the sample is definitely not at the transient adiabatic status due to the significant temperature gradient in the region adjacent to its surface.

Figure 6 also clearly demonstrates the trend of an increase in the CPT value with a shift in the reference point from the sample surface to its interior. These series of CPT values determined are in fact the temperatures at the sample centre for various time intervals, and the highest one corresponds to the status when identical temperatures appear in the central neighborhood. When the highest CPT is reached at the sample centre, it turns to be the peak temperature across the sample radius. As a result, the sample centre 
tends to output heat to the surroundings. This further confirms the sole value of the 'true' CPT at which the heat conduction term vanishes in the central elementary volume.

Consistent with the issue of the presence of thermal conduction term in the energy balance at the CPTs determined by Jones' method, Chen and co-workers pointed out that poor linearity could be found between the logarithmic rates of temperature rise at the crossing point and the reciprocal of the CPTs determined using Jones' approach [17]. Nevertheless, this may not be straight forward in practice. As we can see in Fig. 5 , the ratio $q_{s} / q_{t}$ is getting to $\sim 0.8$ using the Jones' approach, which implies the magnitude of the thermal conduction term of $\sim 0.2 q_{t}$. In other words, the contribution of the term $q_{c}$ may not be considerable in the logarithmic plot of the specific energy terms due to its smaller values at the CPTs. Therefore, the logarithmic plots of the enthalpy change term against the heat generation term at the CPTs should also display acceptable linearity, which has been confirmed in testing [8-12]. Further observations on the ratio of $q_{s} / q_{t}$ at the Jones' CPTs also indicate that it essentially maintains at the same level for various oven temperatures. Thus, the imbalance between the enthalpy change term and the heat generation term at the Jones' CPTs may not make an obvious impact to the slope of the regression line in the plot of $\ln \left(\partial T_{p} / \partial t\right)$ versus $1 / T_{p}$. That is the reason why the value of activation energy obtained using the Jones' approach may be close to that by Chen's method.

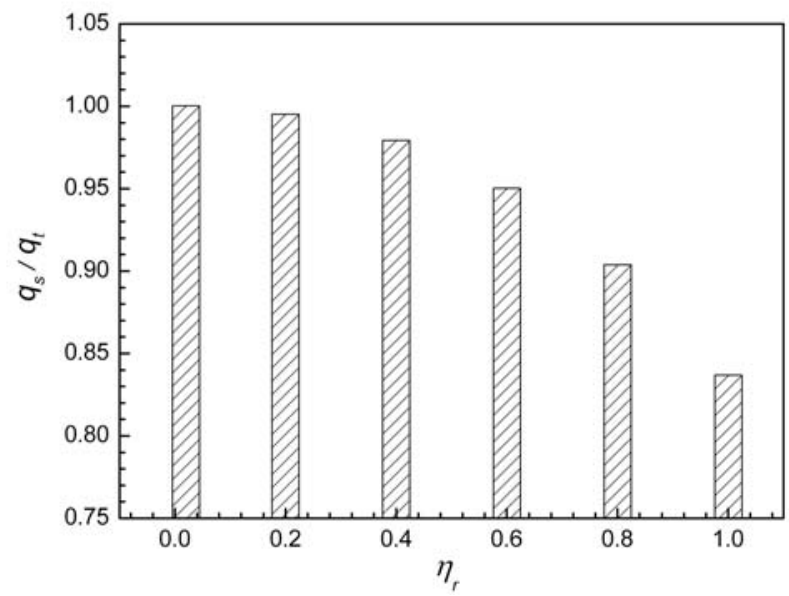

Fig. 5. Effect of non-dimensional distance of the reference point to the sample centre on the ratio $q_{s} / q_{t}$ at the crossing-point temperature for the Solid B with sample radius of $15 \mathrm{~mm}$. The oven temperature was set at $410 \mathrm{~K}$.

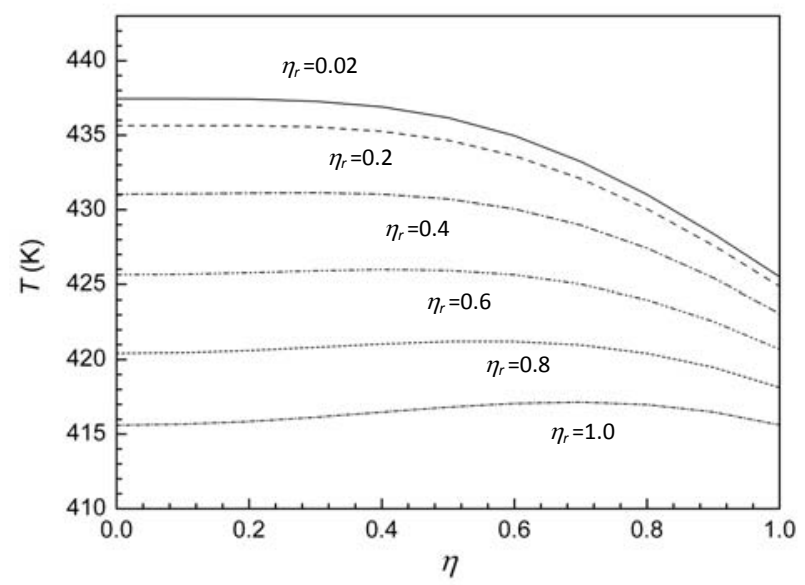

Fig. 6. Temperature profile at the times when identical temperatures are reached between the sample centre and a reference point at varying distances. The parameters' evaluation is the same as that in Fig. 5. 


\section{Possible Positions for Performing Reliable CPT Measurements}

As the position of the reference point departs from the sample centre, the gap between the value of the heat generation term and that of the enthalpy change term at the CPT becomes larger, indicating the presence of the heat conduction term (refer to Fig. 5). This implies the possible inaccuracy in determining the kinetic parameters using a CPT technique. However, at the condition of $0 \leq \frac{q_{t}-q_{s}}{q_{t}} \leq 0.01$, the errors in finding $E$ and $Q A$ are less than $0.1 \%$ and $2.1 \%$, respectively, which is acceptable. Thus, the criterion $q_{s} / q_{t}=0.99$ can be used to determine the maximum distance $r_{m}$ for placing the reference temperature sensor in order to perform a reliable CPT measurement. Figures $7 \mathrm{a}$ and $\mathrm{b}$ show how the dimensionless maximum distance $\eta_{m}$ changes with the sample size for a solid with specific reactivity being heated at a set oven temperature. It is evident that the parameter $\eta_{m}$ increases as the sample size becomes bigger, which is more evident for the solid having a higher activation energy. Lift in the oven temperature results in a slight increase in the dimensionless maximum distance $\eta_{m}$ in most of the cases.

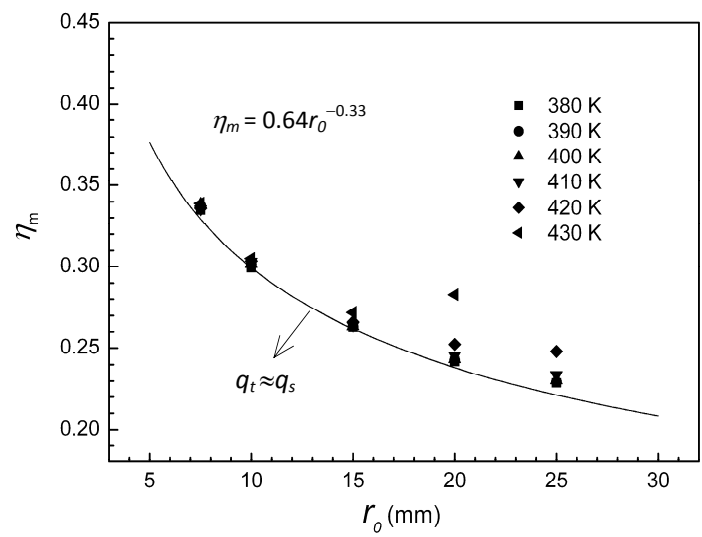

(a)

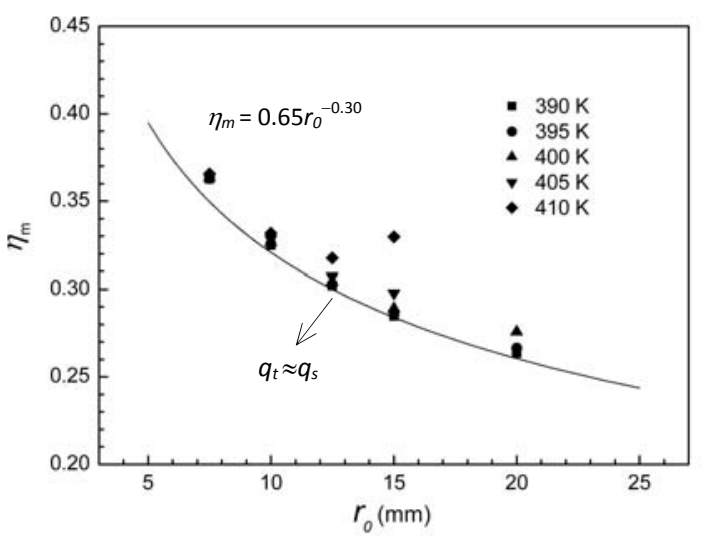

(b)

Fig. 7. Maximum distance determined for placing the reference temperature sensor as a function of sample radius. Calculations were done with both (a) Solid A; (b) Solid B.

The parameter $\eta_{m}$ is apparently a function of several factors including the sample physical and chemical properties as well as the experimental settings, and it is not possible to correlate it with all these factors/variables. However, as shown in Figs. 7a and b, reliable CPT measurements should be achieved once the distance of the reference point meets the condition $\eta_{m} \leq 0.64 r_{0}^{-0.33}$ for the solid having a lower activation energy, and $\eta_{m} \leq 0.65 r_{0}^{-0.30}$ for the solid owning a higher activation energy. By a combination of these results, the following formula is developed for the maximum distance $r_{m}$

$r_{m}=0.64 r_{0}^{0.67}$

where both $r_{0}$ and $r_{m}$ have a unit of $\mathrm{mm}$. Note that this correlation is essentially obtained at the following conditions: the apparent activation energy of the exothermic reactions of a solid ranging from 90.0 to 125.1 $\mathrm{kJ} / \mathrm{mol}$, the reactor diameter $\left(2 r_{0}\right)$ between 15 and $50 \mathrm{~mm}$, and the oven temperature between 380 and 430 $\mathrm{K}$.

The distances constrained by this correlation for various sample sizes coincide with those adopted by three independent groups in their CPT measurements. In the CPT measurements on various types of milk powders, Chong and co-workers [6] set the reference temperature sensor at the distance $4 \mathrm{~mm}$ away from the sample centre for the cylindrical reactor with a diameter of $40 \mathrm{~mm}$, and $5 \mathrm{~mm}$ for the reactor with a dimension of $60 \mathrm{~mm}$. In comparison, the maximum distance determined by Eq. 13 is 4.8 and $6.2 \mathrm{~mm}$, respectively, for the reactors with these two selected dimensions. During the CPT testing with the $0.5 \mathrm{M}$ 
KAc-ion-exchanged coal samples, Zhang and Sujanti [14] placed the reference point at the distance $3 \mathrm{~mm}$ away from the sample centre for the reactor size of $25 \mathrm{~mm}$, which is close to the value $3.5 \mathrm{~mm}$ as determined by Eq. 13. In the assessment on the reactivity of a few types of coal samples, Nugroho and co-authors [13] chose the position of the reference temperature sensor at a distance $5 \mathrm{~mm}$ away from the sample centre, for the reactor with a size of $50 \mathrm{~mm}$, which is also within the maximum distance predicted by Eq. 13 (i.e. $5.5 \mathrm{~mm}$ ).

The reliability of determined maximum distances was further verified here by examining the performance of the CPTs and the corresponding time derivates in finding the parameters $E$ and $Q A$. For the specific solid considered, the CPTs were evaluated for various oven temperatures using a reference point set either adjacent to the centre (i.e. $\eta_{r}=0.02$ ) or at the maximum distance (i.e. $5.5 \mathrm{~mm}$ or $\eta_{r}=0.22$ ), and then their performance was tested in the plot of $\ln \left(\partial T_{p} / \partial t\right)$ against $1 / T_{p}$. As shown in Fig. 8, the results not only exhibit good linearity, but also coincide with each other as well as the experimental data.

Although the formula of the maximum distance was obtained for a long cylindrical reactor, it should also be applicable to a cubical reactor. This is because the determination of the maximum distance was based on the criterion $q_{s} / q_{t}=0.99$, and for a cubical reactor with the shape factor $j=0$, the portion of the heat conduction term in the energy conservation equation decreases in comparison to the reactor owning a shape factor $j=1$. In other words, the criterion for finding the maximum distance also applies to the cubical reactors. In addition, Eq. 13 is only suitable for the reactors with moderate sizes. If the size of a reactor is large enough, the sample may easily reach the super-critical status $[1,2,12,22]$. When this happens, a sharp temperature rise may be presented somewhere between the sample surface and the centre, which leads to the difficulty in finding the CPT prior to the commencement of spontaneous combustion. Under this circumstance, the reliability of a CPT measured and the accuracy in finding the corresponding rate of temperature rise could be in question [12].

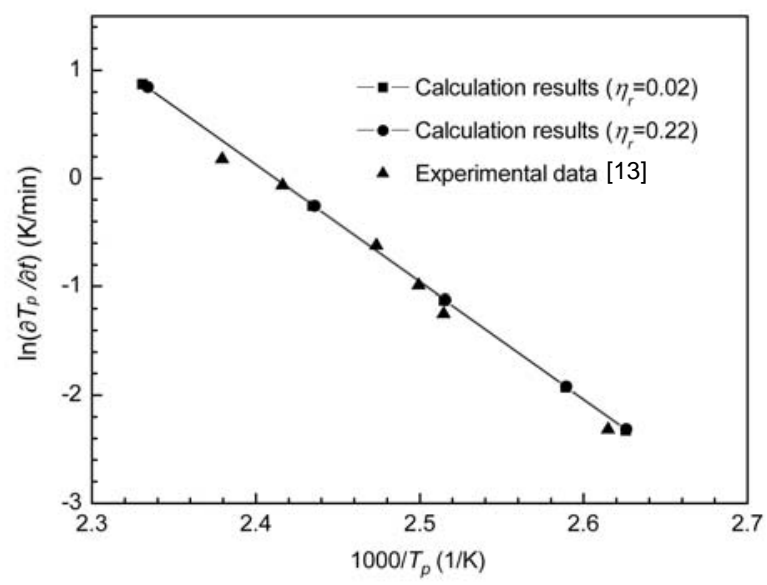

Fig. 8. Plot of $\ln \left(\partial T_{p} / \partial t\right)$ against $1000 / T_{p}$ for the results collected after the reference point was placed at the distance of $0.02 r_{0}$ or $0.22 r_{0}$ (i.e. $5.5 \mathrm{~mm}$ ) individually to the sample centre. The calculations were done for the Solid A with a sample radius of $25 \mathrm{~mm}$.

\section{CONCLUDING REMARKS}

Based upon the energy conservation equation, the time interval when the thermal conduction term vanishes at the centre was traced theoretically for a sample undergoing self-heating at a constant ambient temperature. The central temperature at this time interval, i.e. the true crossing-point temperature, is the transient peak temperature across the sample radius, leading to an overturn in the direction of heat conduction occurring in the central neighborhood. The appearance of a true CPT at the sample centre only occurs once during the self-heating and spontaneous combustion of the sample. 
Analysis indicates that the CPT and its associated time derivative are essentially dominated by two major parameters, i.e. the apparent activation energy of the exothermic reactions and the oven temperature. This finding further confirms the reliability of the CPT techniques in determining the apparent activation energy of exothermic reactions taking place during the self-heating process of a solid, and hence the propensity of a solid to spontaneous combustion.

Calculations have also shown that, with the increase in the distance for placing a reference temperature sensor to find a CPT, the errors for determining the parameters $E$ and $Q A$ become larger, especially for the term $Q A$. At a CPT the enthalpy change term may no longer be equal to that of the heat generation term in the central elementary volume, if the position of the reference temperature sensor is departed from the central neighborhood. However, this trend is somehow minimized at conditions of a reduced sample size and a lower oven temperature.

Within the range of the oven temperatures and experimental sample sizes considered, the maximum distance for placing a reference temperature sensor to achieve a reliable CPT measurement was found to follow a simple correlation $r_{m}=0.64 r_{0}^{0.67}$. Under the experimental conditions considered, this correlation is applicable to a sample either in a cylindrical shape or a square block shape.

\section{REFERENCES}

[1] Bowes, P.C., Self-heating: Evaluating and Controlling the Hazards, Elsevier Press, Amsterdam, the Netherlands, 1984, pp. 17-70.

[2] Babrauskas, V., Ignition Handbook, Fire Science Publishers, Issaquah, WA, 2003, pp. 234-351.

[3] Wang, H.-H., Dlugogorski, B.Z., and Kennedy, E.M., "Tests for Spontaneous Ignition of Solid Materials", Flammability Testing of Materials in Construction, Transport and Mining Sectors, Apte V. (ed.), Woodhead Publishing, Cambridge, UK, 2006, pp. 385-442.

[4] Chen, X.-D., and Chong, L.-V., (1995) Some Characteristics of Transient Self-heating inside an Exothermically Reactive Porous Solid Slab. Trans. IChemE 73(B): 101-107.

[5] Chong, L.-V., Shaw, I.R., and Chen, X.-D., (1995) Thermal Ignition Kinetics of Wood Shavings Measured by a Newly Devised Experimental Technique. Process Safety Progress 14(4): 266-270, http://dx.doi.org/10.1002/prs.680140409

[6] Chong, L.-V., Shaw, I.R., and Chen, X.-D., (1996) Exothermic Reactivities of Skim and Whole Milk Powders as Measured Using a Novel Procedure, Journal of Food Engineering 30: 185-196, http://dx.doi.org/10.1016/S0260-8774(96)00043-X

[7] Chen, X.-D., (1999) On Basket Heating Methods for Obtaining Exothermic Reactivity of Solid Materials: The Extent and Impact of the Departure of the Crossing-point Temperature from the Oven Temperature, Trans. IChemE 77(B): 187-192, http://dx.doi.org/10.1205/095758299530053

[8] Jones, J.C., Chiz, P.S., Koh, R., and Matthew, J., (1996) Continuity of Kinetics between Sub- and Supercritical Regimes in the Oxidation of a High-volatile Solid Substrate. Fuel, 75(15): 1733-1736, http://dx.doi.org/10.1016/S0016-2361(96)00150-0

[9] Jones, J.C., Chiz, P.S., Koh, R., and Matthew, J., (1996) Kinetic Parameters of Oxidation of Bituminous Coals from Heat-release Rate Measurements. Fuel 75(15): 1755-1757, http://dx.doi.org/10.1016/S0016-2361(96)00159-7

[10] Jones, J.C., Henderson, K.P., Littlefair, J., and Rennie, S., (1998) Kinetic Parameters of Oxidation of Coals by Heat-release Measurement and Their Relevance to Self-heating Tests. Fuel 77(1/2): 19-22, http://dx.doi.org/10.1016/S0016-2361(97)00155-5

[11] Jones, J.C., and Puignou, A., (1998) On the Thermal Ignition of Wood Waste. Trans. IChemE 76(B): 205-210, http://dx.doi.org/10.1205/095758298529498 
[12] Nugroho, Y.S., McIntosh, A.C., and Gibbs, B.M., (1998) Using the Crossing Point Method to Assess the Self-heating Behavior of Indonesian Coals. Proceedings of the Combustion Institute, 27: 2981-2989, http://dx.doi.org/10.1016/S0082-0784(98)80158-6

[13] Nugroho, Y.S., McIntosh, A.C., and Gibbs, B.M., (2000) Low-temperature Oxidation of Single and Blended Coals. Fuel 79: 1951-1961, http://dx.doi.org/10.1016/S0016-2361(00)00053-3

[14] Zhang, D.-K., and Sujanti, W., (1999) The Effect of Exchangeable Cations on Low-temperature Oxidation and Self-heating of a Victorian Brown Coal. Fuel 78: 1217-1224, http://dx.doi.org/10.1016/S0016-2361(99)00036-8

[15] Watanabe, W.S., and Zhang, D.-K., (2001) The Effect of Inherent and Added Inorganic Matter on Low-temperature Oxidation Reaction of Coal. Fuel Processing Technology 74: 145-160, http://dx.doi.org/10.1016/S0378-3820(01)00237-5

[16] Chen, X.-D., (2000) Letter to the Editor. Fuel 79: 855-857, http://dx.doi.org/10.1016/S0016-2361(99)00241-0

[17] Chen, X.-D., (2000) Letter to the Editor. Fuel 79: 861, http://dx.doi.org/10.1016/S0016-2361(99)00243-4

[18] Chen, X.-D., and Jones, J.C., (2000) Correspondence, Trans. IChemE 78(B): 68-72, http://dx.doi.org/10.1205/095758200530349

[19] Jones, J.C., (2000) Letter to the Editor. Fuel 79: 859, http://dx.doi.org/10.1016/S0016-2361(99)00242-2

[20] Zhou, L.-X., Theory and Numerical Modeling of Turbulent Gas-particle Flows and Combustion, Science Press \& CRC Press, Beijing, 1993, pp. 36-64. (in Chinese)

[21] Griffiths, J.F., Hasko, S.M., and Tong, A.W., (1985) Thermal Ignition in Packed Particulate Solids: Criticality under Conditions of Variable Biot Number, Combustion and Flame 59: 1-9, http://dx.doi.org/10.1016/0010-2180(85)90052-5

[22] Gray, B.F., Little, S.G., and Wake, G.C., (1992) The Prediction of a Practical Lower Bound for Ignition Delay Times and a Method of Scaling Times-to-Ignition in Large Reactant Masses from Laboratory Data-II. Proceedings of the Combustion Institute, 24: 1785-1791. http://dx.doi.org/10.1016/S0082-0784(06)80209-2 